\title{
Real-world data reveal a diagnostic gap in non-alcoholic fatty liver disease
}

\author{
Myriam Alexander ${ }^{1}$, A. Katrina Loomis ${ }^{2}$, Jolyon Fairburn-Beech ${ }^{1}$, Johan van der Lei ${ }^{3}$, Talita Duarte-Salles ${ }^{4}$, \\ Daniel Prieto-Alhambra ${ }^{5}$, David Ansell ${ }^{6}$, Alessandro Pasqua ${ }^{7}$, Francesco Lapi ${ }^{7}$, Peter Rijnbeek ${ }^{3}$, Mees Mosseveld ${ }^{3}$, \\ Paul Avillach ${ }^{8}$, Peter Egger ${ }^{1}$, Stuart Kendrick', Dawn M. Waterworth', Naveed Sattar ${ }^{9 \dagger}$ \\ and William Alazawi ${ }^{10^{*+}}$ (1)
}

\begin{abstract}
Background: Non-alcoholic fatty liver disease (NAFLD) is the most common cause of liver disease worldwide. It affects an estimated 20\% of the general population, based on cohort studies of varying size and heterogeneous selection. However, the prevalence and incidence of recorded NAFLD diagnoses in unselected real-world healthcare records is unknown. We harmonised health records from four major European territories and assessed ageand sex-specific point prevalence and incidence of NAFLD over the past decade.
\end{abstract}

Methods: Data were extracted from The Health Improvement Network (UK), Health Search Database (Italy), Information System for Research in Primary Care (Spain) and Integrated Primary Care Information (Netherlands). Each database uses a different coding system. Prevalence and incidence estimates were pooled across databases by random-effects metaanalysis after a log-transformation.

Results: Data were available for 17,669,973 adults, of which 176,114 had a recorded diagnosis of NAFLD. Pooled prevalence trebled from $0.60 \%$ in 2007 (95\% confidence interval: $0.41-0.79)$ to $1.85 \%(0.91-2.79)$ in 2014. Incidence doubled from $1.32(0.83-1.82)$ to 2.35 (1.29-3.40) per 1000 person-years. The FIB-4 non-invasive estimate of liver fibrosis could be calculated in $40.6 \%$ of patients, of whom $29.6-35.7 \%$ had indeterminate or high-risk scores.

Conclusions: In the largest primary-care record study of its kind to date, rates of recorded NAFLD are much lower than expected suggesting under-diagnosis and under-recording. Despite this, we have identified rising incidence and prevalence of the diagnosis. Improved recognition of NAFLD may identify people who will benefit from risk factor modification or emerging therapies to prevent progression to cardiometabolic and hepatic complications.

Keywords: Epidemiology, Population, NAFLD, NASH

\section{Background}

Non-alcoholic fatty liver disease (NAFLD) is rapidly becoming the most common cause of chronic liver disease worldwide [1]. NAFLD is a spectrum of diseases that encompasses uncomplicated steatosis, non-alcoholic steatohepatitis (NASH) and fibrosis, which in a small proportion can lead to complications including cirrhosis, liver failure and hepatocellular carcinoma [2]. NAFLD is a multisystem disease with a multidirectional relationship

\footnotetext{
* Correspondence: w.alazawi@qmul.ac.uk

${ }^{\dagger}$ Naveed Sattar and William Alazawi contributed equally to this work.

${ }^{10}$ Barts Liver Centre, Blizard Institute, Queen Mary, University of London, London, UK

Full list of author information is available at the end of the article
}

with the metabolic syndrome [3-5]. NAFLD is associated with increased risk of cardiovascular disease [5-7] and cancer [8]. Among other high-risk groups [9], people with diabetes and NAFLD are at increased risk of micro- and macrovascular complications $[10,11]$ and these patients have a twofold increased risk of all-cause mortality [12].

The estimated point prevalence of NAFLD in the general Western population is $20-30 \%$, largely based on cohort studies with heterogeneous inclusion criteria and research methods [13]. The prevalence of NAFLD rises to $40-70 \%$ among patients with type 2 diabetes and up to $90 \%$ among patients with morbid obesity [14-16]. Moreover, as the rates of diabetes and obesity rise worldwide, it is expected that NAFLD will become even more

(c) The Author(s). 2018 Open Access This article is distributed under the terms of the Creative Commons Attribution 4.0 International License (http://creativecommons.org/licenses/by/4.0/), which permits unrestricted use, distribution, and 
common. NAFLD-related cirrhosis is currently the third most common indication and is anticipated to become the leading indication for liver transplantation in the USA within the next one to two decades [17].

There is much debate about whether screening programmes in the general population or in at-risk groups, such as people with diabetes [9], should be implemented $[18,19]$. This debate is based on our current understanding of the epidemiology and natural history of NAFLD, which, in turn, derives from cohort or cross-sectional studies [13]. These are often highly selected studies of individuals with metabolic risk factors, or they involve extensive phenotyping that would be unrealistic in routine practice.

A pragmatic approach is to focus on real-world patients for whom the diagnosis of NAFLD has been made during routine clinical care. A diagnosis of NAFLD is often made following abnormal imaging of the liver or elevated serum liver enzymes (so-called liver function tests) and involves exclusion of other causes of liver injury, such as excess alcohol consumption and viral hepatitis. Although routinely collected data can represent only the visible part of the clinical iceberg, there is a growing body of literature that has used well-curated electronic health records (EHRs) to study disease characteristics and epidemiology in large numbers of people [20-22].

In many European countries where health care is largely state funded and there are low or absent primary-care co-payments, the population has unrestricted access to health care with primary-care physicians acting as gatekeepers (including referral to secondary care) [23]. Healthy people register with primary-care centres when they move to an area to access health care when it is be needed and so primary-care EHR represent data that are as close to a general population as possible, with near universal coverage of the population in the region where the data is collected. Recording of a diagnosis in European primary-care databases is not driven by reimbursement and the patient population is relatively stable compared to other types of EHRs, such as US claims databases. Primary-care databases hold comprehensive medical records, which include diagnoses, prescriptions, laboratory values, lifestyle and health measures, and demographic information for a large and representative sample of patients. Concerns around the degree of data completeness are now largely historic as the vast majority of practices are paper-free and therefore, these data represent the only clinical record for care, administration and re-imbursement. Thus, within the areas that utilise these databases, coverage is near universal. If a practice joins the database, all the patients of that practice are registered in the database. Although there is an option for individual patients to opt out, this is minimal $(<1 \%)$.
In this study, we harmonised health-care records for 17.7 million adults from four large European primary-health-care databases to estimate the prevalence and incidence of recorded diagnoses of NAFLD and, where available, $\mathrm{NASH}$, in patients in primary care and to compare these with estimates from cohort studies. We sought to ascertain the changes in prevalence and incidence of recorded diagnoses of NAFLD from 2007 to 2015 , and the effect of age and sex. We compared the characteristics of patients with an NAFLD diagnosis in the different databases and reported, where possible, the proportion of patients with markers of advanced disease in the diagnosed population.

\section{Methods}

\section{Databases}

Ethical approval was obtained by data custodians of each primary-care database according to local institutional review board requirements. Anonymised data were extracted from the Health Search Database (HSD) in Italy [24], the Integrated Primary Care Information (IPCI) in the Netherlands [25], The Health Improvement Network (THIN) in the UK [26] and the Information System for Research in Primary Care (SIDIAP) in the Catalonia region of Spain [27] (Additional file 1: Table S1).

THIN, HSD and IPCI had all reached high levels of patient registration from January 2004 onwards. SIDIAP started data collection in 2005 and has high quality data from 2006. Data entered between 1 January 2004 (SIDIAP from 1 January 2007) and up to 31 December 2015 were included in incidence estimates. Individuals were excluded if they had less than 1 year of follow-up post registration into the database. Individuals with a diagnosis of NAFLD were not included in the analyses if they also had a recorded history of alcohol abuse. To maximise data completeness, we included only patients whose NAFLD diagnosis occurred within \pm 6 months of a general practitioner (GP) visit when describing patients' characteristics (Table 1 and Additional file 1: Table S3).

\section{Patient involvement}

All eligible patients were included in the study. Routine health-care records were collected from patients at each encounter with a health-care practitioner. Following local regulations, patients who did not wish to share their data were able to withdraw from the databases.

\section{Semantic harmonisation and case ascertainment}

The four databases each use different coding systems (Additional file 1: Table S1). As a result, the capture of NAFLD and NASH diagnoses differed across databases. In HSD and IPCI, NAFLD and NASH were captured in a single code as 'NAFLD or NASH'. In SIDIAP and THIN, NAFLD and NASH were coded separately, 
Table 1 Flow chart of identification of NAFLD patients

\begin{tabular}{llllll}
\hline Flow chart & HSD (Italy) & IPCI (Netherlands) & SIDIAP (Spain) & THIN (UK) & Total \\
\hline $\begin{array}{l}\text { Total number of individuals ever enrolled } \\
\text { by December } 2015\end{array}$ & $1,571,651$ & $2,225,925$ & $5,488,397$ & $12,695,046$ & $21,981,019$ \\
$\begin{array}{l}\text { Number of individuals with } \geq 1 \text { year of } \\
\text { registration in adulthood }\end{array}$ & $1,544,573$ & $1,780,500$ & $5,259,575$ & $9,085,325$ & $17,669,973$ \\
$\begin{array}{l}\text { Number of NAFLD patients } \\
\text { NAFLD: 27,002 }\end{array}$ & $\begin{array}{l}\text { NAFLD: 48,036 (19,048 were } \\
\text { incident post IPCI starting date) }\end{array}$ & $\begin{array}{l}\text { NAFLD: 77,547 } \\
\text { NASH only: 1887 }\end{array}$ & $\begin{array}{l}\text { NAFLD: 23,529 } \\
\text { NASH only: 1133 }\end{array}$ & NAFLD: 176,114 \\
\hline
\end{tabular}

The descriptive tables (Table 2 and Additional file 1: Table S3) include only patients with an incident diagnosis made within the study period and with a record of a GP visit within \pm 6 months of diagnosis. Numbers for NAFLD/NASH were as follows: HSD 24,027, IPCI 18,865, SIDIAP 77,107 and THIN: 12,385 individuals. Note in HSD and IPCI, 'NAFLD' is likely to include patients with NASH since no separate term for NASH exists in these databases. The number in the 'Total' column includes patients within SIDIAP and THIN who have NASH

GP general practitioner, HSD Health Search Database, IPCI Integrated Primary Care Information, NAFLD non-alcoholic fatty liver disease, NASH non-alcoholic steatohepatitis, SIDIAP Information System for Research in Primary Care, THIN The Health Improvement Network

branching out of a 'NAFLD or NASH' code. In this study, we extracted all 'NAFLD or NASH' diagnoses as well as 'NASH only diagnoses' where available. For simplicity, we labelled 'NAFLD or NASH' as 'NAFLD' and 'NASH only' as 'NASH'. Code lists were generated for the four terminologies (ICD9CM, Read Codes, SNOMEDCTUS and ICD10) that mapped to the same Unified Medical Language System (UMLS) concepts [28] (Additional file 1: Table S2).

Clinical diagnoses were defined with these code lists using the same process of harmonisation (code lists available on request). In SIDIAP, we used a combination of clinical codes and answers to questionnaires on alcohol consumption to identify alcohol abuse.

Given the absence of a code for NAFLD in the IPCI terminology, we additionally used text-mining in this database. The algorithm to identify NAFLD in IPCI is detailed in Additional file 1: Figure S1. Patients with records for the following search terms were extracted: 'NASH, 'NAFLD', 'steatohepatitis' or 'fatty liver disease' as distinct words preceded by a space and followed by a space, or at the beginning or end of a sentence. Patients with relevant search terms preceded by a negation term (e.g. 'no' or 'not') were excluded. To validate the text-mining, 100 individuals identified using free-text were randomly sampled. Their complete medical charts were manually reviewed to confirm that the clinical data support the text-mining-derived diagnosis.

\section{Use of historical data}

Governance rules differed between the different databases. In HSD and SIDIAP, there were no records available prior to a primary-care practice joining the database. In THIN, data from patients who had already left the practice were available, so NAFLD/NASH diagnoses made prior to the patient's primary-care practice joining THIN were counted in both incidence and prevalence estimates. However, in IPCI, records that predated their primary-care practice joining the database were available only for patients who remained in the practice (since leavers did not have the opportunity to refuse to participate). Therefore, historic diagnoses could be included in the point prevalence. However, given that both the number of new diagnoses made as well as the total number of patients at risk in a given period were unknown, we could not include diagnoses made before the patient joined a practice in incidence estimates in IPCI.

\section{Other data extraction}

Demographic information, lifestyle and medical history of relevant morbidities were also extracted for all NAFLD and NASH patients identified in the four databases. Medical records for type 2 diabetes and hypertension at any time prior to NAFLD or NASH diagnosis were extracted. Code lists for those diagnoses were harmonised across the databases using the semantic harmonisation described in 'Methods', which aligns all terms for the same list of UMLS concepts (code lists available on request).

Laboratory values for aspartate transaminase (AST), alanine transaminase (ALT) and platelet count were extracted. We used the values closest to the time of NAFLD diagnosis (up to 2 years prior to diagnosis or less than 6 months after). Body mass index (BMI) was calculated for all NAFLD patients with weight recorded between 2 years prior to and 6 months after diagnosis, and with height recorded anytime in adulthood. We excluded values that were likely to be implausible: BMI below $15 \mathrm{~kg} / \mathrm{m}^{2}$, laboratory values greater than the mean in the database plus 3 times the standard deviation, AST and ALT less than $5 \mathrm{IU} / \mathrm{L}$, and platelet counts below $5 \times 10^{9} \mathrm{~L}^{-1}$.

The FIB-4 index was calculated to provide an estimate of the severity of fibrosis in patients at the time of their NAFLD diagnosis. The formula for FIB-4 is: Age [years] $\times$ AST $[\mathrm{U} / \mathrm{L}] /\left(\right.$ platelet $\left[10^{9}\right] \times \sqrt{ }$ ALT $\left.[\mathrm{U} / \mathrm{L}]\right)[29]$. The cut-offs for FIB-4 scoring for NAFLD are $<1.30$ for a low risk of advanced fibrosis or cirrhosis, between 1.30 and 2.67 for an indeterminate score and 2.67 for a high risk of advanced fibrosis or cirrhosis [30].

\section{Statistical methods}

Quantitative variables were reported as mean and 95\% confidence interval $(\mathrm{CI})$ of the mean assuming a normal 
distribution, and qualitative variables as percentages. Differences in patients' characteristics between the four databases were tested by an ANOVA test for quantitative characteristics and a chi-square test for categorical characteristics.

Incidence in the adult population aged $\geq 18$ years old was estimated by dividing the number of individuals with a diagnosis of NAFLD (or NASH where relevant) by the total number of person-years at risk. Incidence was reported by predefined age categories, gender and calendar year.

Point prevalence was estimated for 1 January of each calendar year available in the data, by gender and by predefined age categories. Point prevalence was defined as the total number of individuals with a recorded NAFLD diagnosis at or prior to 1 January of a calendar year and who were still active in the database, divided by the total number of active patients in the database on that date.

In addition, the 1-year period prevalence was estimated in a sensitivity analysis to account for potential differences in length of follow-up across databases, and over time within databases. The 1-year period prevalence was defined for each calendar year available as the number of new individuals with a recorded diagnosis of NAFLD in a calendar year divided by the average number of active patients in that year (defined as the number on 1 January plus the number on 31 December divided by 2).

Age was computed at the end of the year for period prevalence (31 December of the year of interest). For point prevalnce, age was computed on 1 January of the year of interest. Within each database, incidence estimates were compared by calendar year (assuming a linear relationship), sex (males are the reference group) and age group (age 60-69 is the reference group) by fitting Poisson distributions. Prevalence estimates were compared by fitting logistic regressions and performing chi-square tests. $P<$ 0.001 was considered as significant, although note that with such large datasets, a high level of significance can be achieved even for minimal absolute differences in prevalence and incidence levels.

Incidence and prevalence estimates were pooled for each calendar year across the four databases using a random effects meta-analysis after natural log-transformation (weighting by the inverse of the variance). We reported the $I^{2}$ statistic, which gives the percentage of variation among databases attributable to heterogeneity, and the $p$ values of heterogeneity ( $\mathrm{p}$-het), tested using $\mathrm{Q}$ statistics. To investigate sources of heterogeneity, we tested for a linear association between incidence and point prevalence with calendar year by fitting a meta-regression.

Data were extracted and analysed using the European Medical Information Framework (EMIF) with a distributed network approach that allows data custodians to maintain control over their protected data [31]. Each data custodian extracted data from their database into four common files: prescriptions, measurements, events and patients. These files were transformed locally by the data transformation tool Jerboa Reloaded, which produces analytical datasets that can be shared with data analysts in a central remote research environment for further post-processing. The analytical datasets contained characteristics for each patient with a NAFLD diagnosis, as well as aggregated results on incidence and prevalence by age, gender and calendar year. Quality controls were run on each database and the research team communicated with data custodians to confirm results. Statistics and graphics were generated in the remote research environment using the statistical software Stata/SE 14.1.

\section{Results}

Semantic harmonisation to identify the European NAFLD cohort

In total, the four European databases held data on $21,981,019$ patients, of whom 17,699,973 adults had been registered for at least 1 year in adulthood (Table 1). Using semantic harmonisation, we identified 176,114 patients who had a recorded diagnosis of NAFLD (including NASH). This represents $1.0 \%$ of the total population, ranging from $0.3 \%$ in the UK (THIN) to $2.7 \%$ in the Netherlands (IPCI). The largest number of NAFLD patients was in the Spanish cohort (SIDIAP, $n=77,547$, Table 1). Recording of NASH diagnoses was possible only in Spain (SIDIAP, $n=1887$ ) and in the UK (THIN, $n=1133$ ), as the other two databases did not have specific codes distinguishing NAFLD from NASH. Given the small numbers overall, we did not pursue an analysis of NASH incidence and prevalence further and we included these patients within the total number of patients with a recorded diagnosis of NAFLD.

In the Dutch database (IPCI), the majority of patients were identified via free-text mining with seed words 'NAFLD', 'NASH,' 'fatty liver' or 'steatosis', and a minority from diagnostic codes only (see Additional file 1: Figure S1). The code for 'liver steatosis' (D97.05) identified 1282 patients. The code for 'cirrhosis/other liver disease' (D97.00) identified 4228 patients when combined with a free-text search on the code label and 1214 additional patients when combined with a free-text search anywhere in the medical records. Searching for the search terms in free text in the absence of a relevant code identified 44,442 additional patients. Of these, 19,048 patients had an incident NAFLD diagnosis (recorded at a time when the patient's general practice was contributing to IPCI). In the sample of 100 cases that were manually reviewed, the positive predictive value for a text-mined diagnosis of NAFLD was $98 \%$. 
We identified only a small proportion of patients with a recorded diagnosis of NAFLD who also drank alcohol in excess of recommended limits: 3130 (7.0\%) NAFLD patients in IPCI, 921 in HSD (3.3\%), 12,461 in SIDIAP (14.1\%) and 925 in THIN (3.8\%). These patients were excluded from the statistical analysis.

The characteristics of the populations of patients with an incident diagnosis of NAFLD made during the study period, after exclusions, are shown in Table 2 for the individual databases. There were minor differences in the mean age, proportion of patients with impaired fasting glucose or diabetes, and platelet count in each of the four databases. However, we observed that HSD had statistically significantly higher proportions of males and patients with hypertension than other databases. There was considerable variation in recorded BMI $\left(29.7 \mathrm{~kg} / \mathrm{m}^{2}\right.$ in HSD to $32.4 \mathrm{~kg} / \mathrm{m}^{2}$ in THIN), alanine transaminase (ALT) levels (median $28 \mathrm{IU} / \mathrm{L}$ in HSD to $39 \mathrm{IU} / \mathrm{L}$ in THIN) and aspartate transaminase (AST) levels (median $24 \mathrm{IU} / \mathrm{L}$ in HSD to $32 \mathrm{IU} / \mathrm{L}$ in THIN). Moreover, we observed variation in clinical practice with higher rates of BMI being recorded and ALT requests in THIN and SIDIAP compared to IPCI and HSD (Table 2 and Additional file 1: Table S3).

Non-invasive scores that estimate the degree of liver fibrosis can be calculated from clinical parameters and are used to risk-stratify patients with NAFLD. Although both ALT and AST are required to calculate the majority of such non-invasive scores, ALT was more frequently available than AST in all four databases (Additional file 1: Table S3). An AST result was available for $21 \%$ (THIN) to 68\% (HSD) and an ALT result for 67\% (IPCI) to 86\% (SIDIAP). This is reflected in the proportion of patients in whom a FIB-4 non-invasive assessment of liver fibrosis could be calculated, ranging from $11 \%$ in THIN to $54 \%$ in SIDIAP. Despite having the smallest number (and percentage) of patients in whom we could calculate FIB-4, the THIN database had the highest proportion of patients with high-risk scores indicative of advanced fibrosis or even cirrhosis $(10.0 \%$ vs $2.9-$ $4.3 \%, p<0.001)$. In practice, patients with indeterminate or high-risk scores are often managed with further assessment leading to a liver biopsy. The proportion of patients with intermediate/high-risk scores was lower in IPCI $(29.8 \%)$ compared to the other databases (35.0-35.7\%); albeit the number of people for whom we could calculate FIB- 4 was variable.

\section{The rising prevalence of NAFLD diagnosis}

The overall (pooled) prevalence of NAFLD diagnosis was low at $1.85 \%$ (95\% CI: 0.91-2.79) $\left(I^{2}=99.99 \%\right.$, p-het $<0.001)$ on 1 January 2015, but it had trebled from $0.60 \%(0.41-0.79) \quad\left(I^{2}=99.97 \%\right.$, p-het $\left.<0.001\right)$ on 1 January 2007 (Fig. 1 and Additional file 1: Table S4).

The prevalence of recorded NAFLD diagnosis rose over time in all databases, albeit levels and rates of rise

Table 2 Descriptive characteristics of patients with an incident diagnosis of NAFLD in four European primary-care databases

\begin{tabular}{|c|c|c|c|c|c|}
\hline \multirow[t]{2}{*}{ Characteristics } & $\mathrm{HSD}(\mathrm{N}=24,027)$ & $\mathrm{IPCI}(N=18,865)$ & $\operatorname{SIDIAP}(N=77,107)$ & THIN $(N=23,385)$ & \multirow[t]{2}{*}{$\begin{array}{l}\text { Test of difference } \\
p \text { value }\end{array}$} \\
\hline & $\begin{array}{l}\% \text { / Mean (SD) / } \\
\text { median (IQR) }\end{array}$ & $\begin{array}{l}\% \text { / Mean (SD) / } \\
\text { median (IQR) }\end{array}$ & $\begin{array}{l}\% \text { / Mean (SD) / } \\
\text { median (IQR) }\end{array}$ & $\begin{array}{l}\% \text { / Mean (SD) / } \\
\text { median (IQR) }\end{array}$ & \\
\hline Age in years & $56.2(14.3)$ & $56.8(13.9)$ & $56.0(13.4)$ & $53.7(13.4)$ & $<0.0001$ \\
\hline Gender (males) & $57.3 \%$ & $49.3 \%$ & $52.7 \%$ & $51.5 \%$ & $<0.0001$ \\
\hline Body mass index in $\mathrm{kg} / \mathrm{m}^{2}$ & $29.7(5.0)$ & $30.8(5.4)$ & $31.3(5.1)$ & $32.4(5.9)$ & $<0.0001$ \\
\hline $\begin{array}{l}\text { History of diabetes or } \\
\text { impaired fasting glucose }\end{array}$ & $18.0 \%$ & $20.5 \%$ & $20.0 \%$ & $21.0 \%$ & $<0.0001$ \\
\hline History of hypertension & $47.5 \%$ & $36.0 \%$ & $42.8 \%$ & $40.5 \%$ & $<0.0001$ \\
\hline Aspartate transaminase (IU/L) & $24(19-33)$ & $29(22-40)$ & $29(22-40)$ & $32(24-47)$ & $<0.0001$ \\
\hline Alanine transaminase (IU/L) & $30(20-48)$ & $37(25-56)$ & $34(22-53)$ & $45(28-68)$ & $<0.0001$ \\
\hline Platelet counts $\left(10^{9} / \mathrm{L}\right)$ & $238(65)$ & $262(68.6)$ & $244(61.2)$ & $250(75.3)$ & $<0.0001$ \\
\hline AST to ALT ratio & $0.87(0.34)$ & $0.80(0.36)$ & $0.83(0.38)$ & $0.82(0.38)$ & $<0.0001$ \\
\hline FIB-4 score & & & & & $<0.0001$ \\
\hline Low risk (FIB-4 < 1.30) & $64.3 \%$ & $70.4 \%$ & $65.5 \%$ & $65.0 \%$ & \\
\hline $\begin{array}{l}\text { Indeterminate risk } \\
\text { (FIB-4: 1.30-2.67) }\end{array}$ & $31.4 \%$ & $26.7 \%$ & $30.3 \%$ & $25.0 \%$ & \\
\hline High risk (FIB-4 > 2.67) & $4.3 \%$ & $2.9 \%$ & $4.2 \%$ & $10.0 \%$ & \\
\hline
\end{tabular}

Arithmetic means were reported for age, BMl, platelet counts and AST to ALT ratio; median (IOR) were reported for albumin, AST and ALT. P values are from ANOVA test of difference between means for continuous variables (for log-transformed AST and ALT), and Chi-2 test of difference for categorical variables. Number of patients with data available on each of these variables is provided in Additional file 1: Table S3

ALT alanine transaminase, ANOVA analysis of variance, AST aspartate transaminase, BMI body mass index, HSD Health Search Database, IPCI Integrated Primary Care Information, IQR interquartile range, SD standard deviation, SIDIAP Information System for Research in Primary Care, THIN The Health Improvement Network 


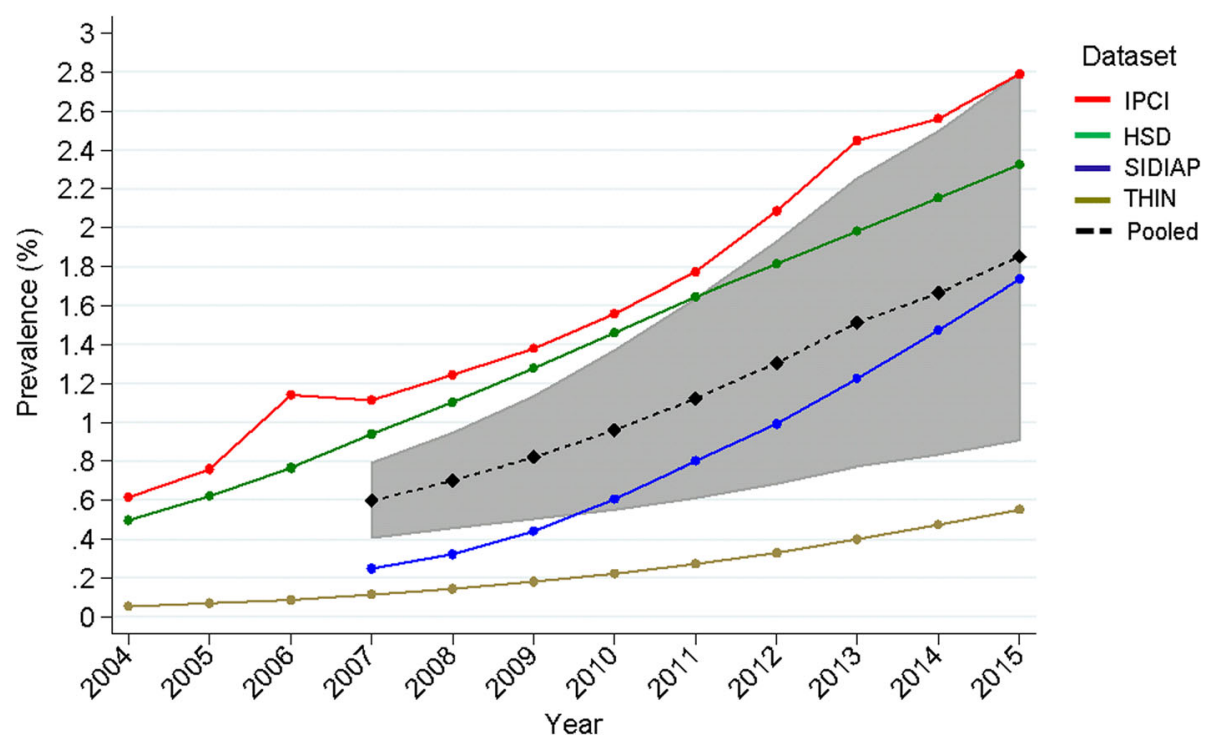

Fig. 1 Point prevalence of NAFLD (per 100 persons) by calendar year. Results are shown for each database and pooled across databases by metaanalysis. The pooled estimate is provided from 2007 only as data from SIDIAP were available only from that year onward. The pooled estimate confidence interval is shaded grey. HSD Health Search Database, IPCI Integrated Primary Care Information, NAFLD non-alcoholic fatty liver disease, SIDIAP Information System for Research in Primary Care, THIN The Health Improvement Network

differed between databases, being highest in the Netherlands (IPCI) and lowest in the UK (THIN). To confirm that those trends were not due to more complete medical records being available in more recent years, we also estimated 1-year period prevalence and observed rising trends for the four databases (Additional file 1: Table S5).

There were no significant differences in prevalence between sexes in any database, but prevalence did vary by age. Peak prevalence was in patients aged 60-79 in whom it was $>20$ times higher than in 18-29 years old in IPCI (4.89\% versus $0.24 \%$ ) and $10-14$ times higher in the other databases (Fig. 2 and Additional file 1: Table S6).

\section{Incidence of NAFLD has doubled since 2007}

The overall (pooled) incidence of recorded NAFLD diagnoses was $2.35\left(1.29-3.40 ; I^{2}=99.92 \%\right.$, p-het $\left.<0.001\right)$ per 1000 person-years in 2015, having approximately doubled since 2007 (1.32; 0.83-1.82)) (see Fig. 3 and Additional file 1: Table S7).

We observed heterogeneity between databases. In IPCI and SIDIAP, there was a clear and consistent rise in incidence with a 2.7-fold increase from 2004 to 2015 to 4.09 per 1000 person-years in IPCI and 3.2-fold increase from 2007 to 2015 to 2.61 per 1000 person-years in SIDIAP. In HSD, there was no statistically significant change in incidence between 2005 and 2015 (Additional file 1: Table S6). Although the rate of rise in THIN was comparable to IPCI and SIDIAP, the very low starting rate meant that despite a fivefold increase, the absolute increase was still modest and the incidence in 2014 was 1.08 per 1000 person-years.
There was a significant difference between sexes in HSD and SIDIAP $(p<0.05)$ but not in IPCI and THIN. In HSD, IPCI and SIDIAP, peak incidence was in 60-69 year olds, and in 50-59 year olds in THIN (but the estimate was not significantly different from that in 60-69 year olds) and then decreased in older age groups (Fig. 4, Additional file 1: Table S8).

\section{Discussion}

In the largest real-world study of its kind to date, we report the incidence and prevalence of recorded NAFLD diagnoses among 17.7 million adults in four different European countries.

The databases used have been validated, are broadly representative of the population of the country and have been extensively used for pharmaco-epidemiology research [17, 20] (Additional file 1: Table S1). Despite a rise in incidence, our study found a large shortfall in Europe between the expected number of patients with NAFLD and NASH and the number with recorded diagnoses. Although others have suggested that this might be the case at a local level or in small questionnairebased exercises [32], this study has identified the scale of that diagnostic gap across four European territories. Under-recording of NAFLD in primary care may reflect (i) missed opportunities to make the diagnosis by investigating abnormal liver enzyme values or imaging findings, (ii) a lack of confidence to make the diagnosis even if liver enzymes are in the reference range or (iii) under-recognition of the diagnosis in secondary care. Furthermore, many patients who do have the diagnosis 


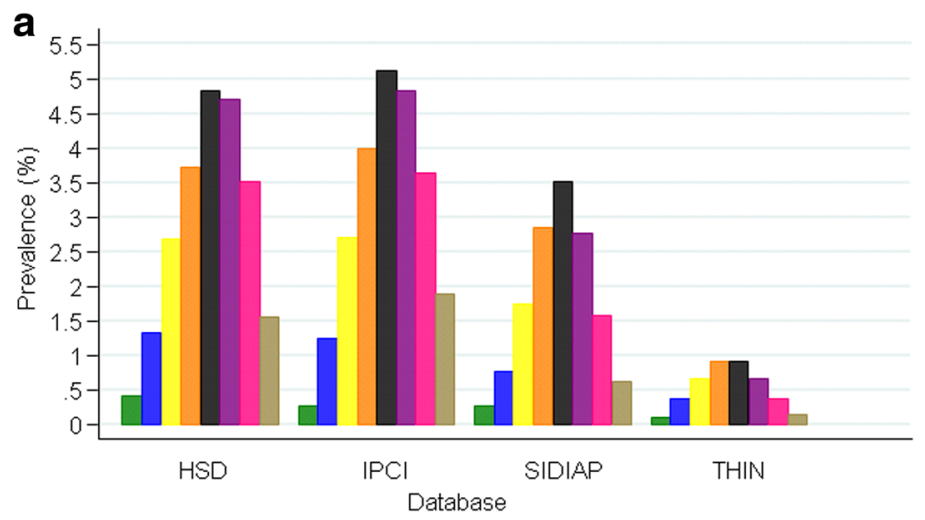

\section{Age groups}

$\begin{array}{r}18-29 \text { years } \\ \hline 30-39 \text { years }\end{array}$

$40-49$ years

50-59 years

60-69 years

70-79 years

80-89 years

$\geq 90$ years

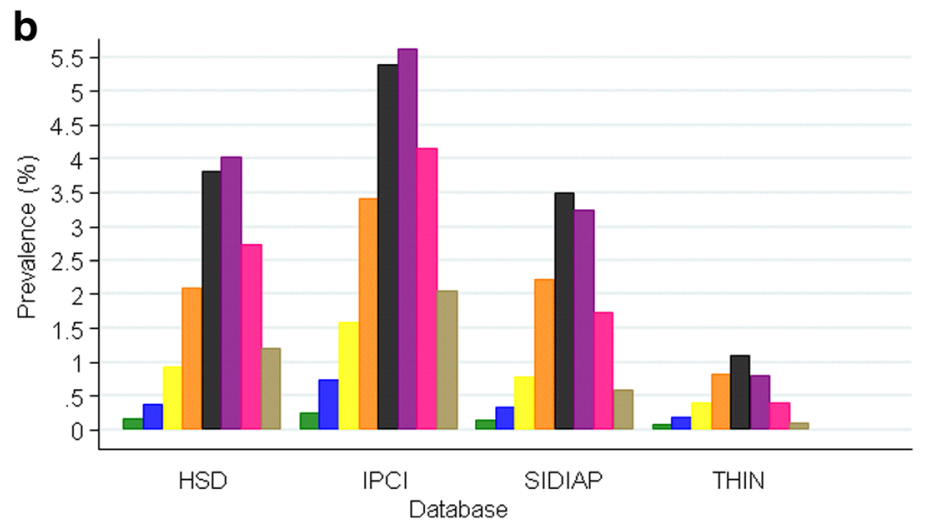

Age groups

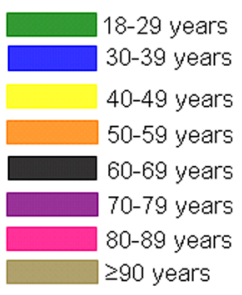

Fig. 2 Point prevalence of NAFLD (per 100 persons) by age group on 1 January 2015 in a males and b females. HSD Health Search Database, IPCI Integrated Primary Care Information, NAFLD non-alcoholic fatty liver disease, SIDIAP Information System for Research in Primary Care, THIN The Health Improvement Network

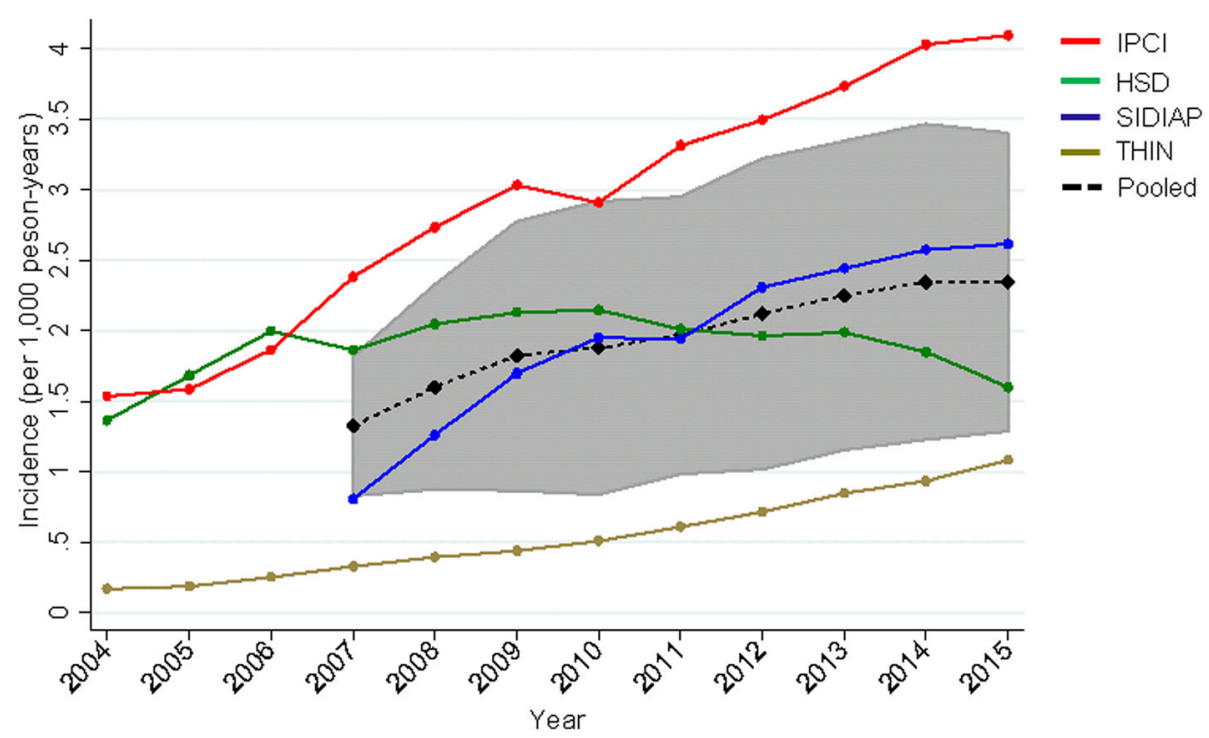

Fig. 3 Incidence of NAFLD (per 1000 person-years) by calendar year in four primary-care databases, and pooled across databases by a random effects meta-analysis. The pooled estimate is provided from 2007 only as data from SIDIAP were available only from that year onward. The pooled estimate confidence interval is shaded grey. HSD Health Search Database, IPCI Integrated Primary Care Information, NAFLD non-alcoholic fatty liver disease, SIDIAP Information System for Research in Primary Care, THIN The Health Improvement Network 

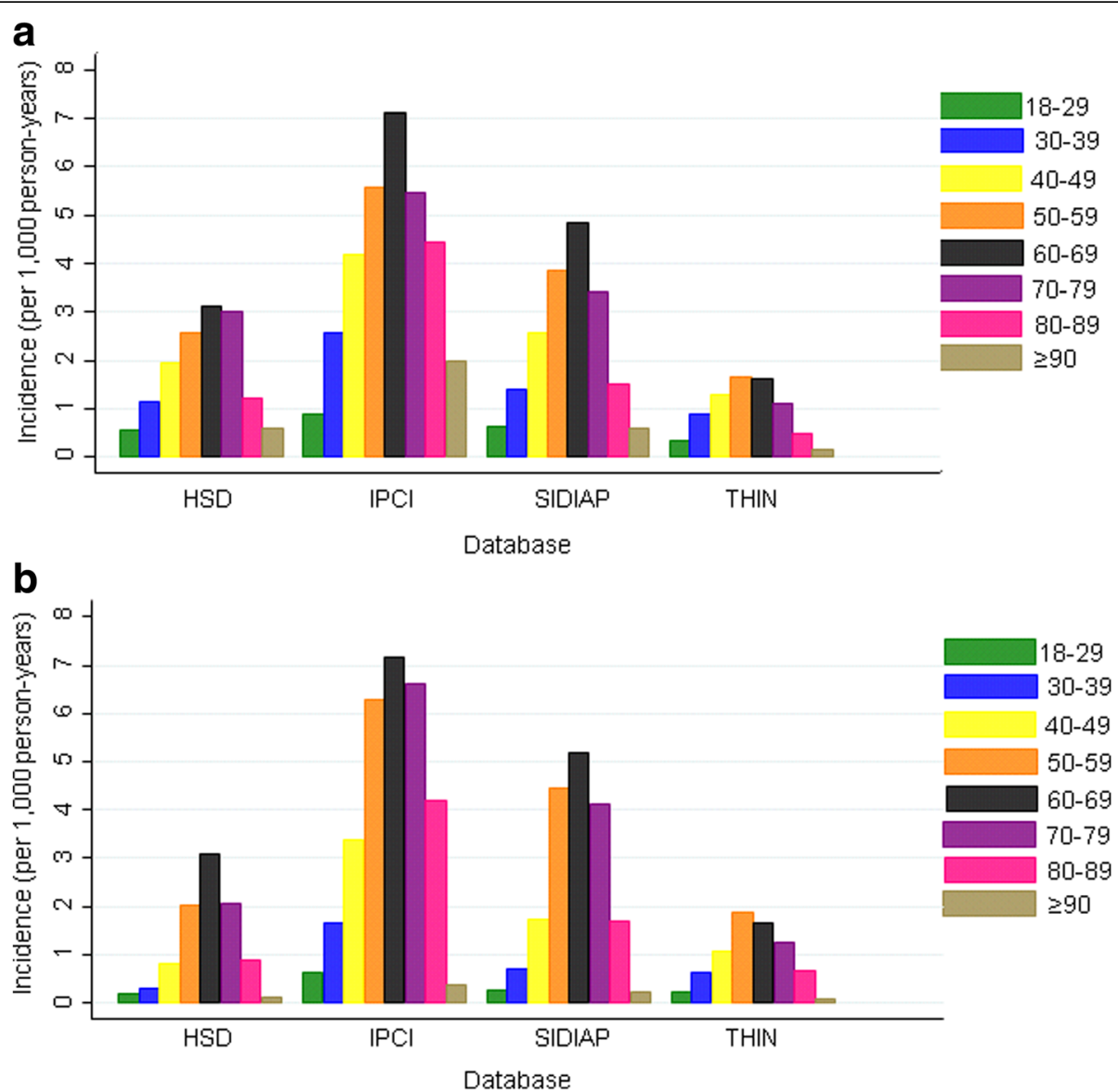

Fig. 4 Incidence of NAFLD (per 1000 person-years) by age group for the four primary care databases for 2015 in a males and $\mathbf{b}$ females. HSD Health Search Database, IPCI Integrated Primary Care Information, NAFLD non-alcoholic fatty liver disease, SIDIAP Information System for Research in Primary Care, THIN The Health Improvement Network

have not had the investigations required for appropriate risk-stratification and therefore, specialist care may not be offered to those at greatest need. The current study represents a departure from existing population-level study designs of NAFLD. Notwithstanding the limitations discussed below, by using real-world data, we have gained insight into current practice and attitudes to NAFLD and into the changing face of NAFLD in primary care.

We used UMLS semantic harmonisation to extract primary-care EHR data and identify 176,114 patients with a recorded diagnosis of NAFLD. Despite variations in coding systems, in the characteristics of the populations and in the health-care systems in each country, the results from all four territories are broadly consistent. They show rising incidence and prevalence of NAFLD; however, the levels of recorded NAFLD in EHR primary-care databases is many-fold lower than those anticipated based on prior observation studies, which estimated the prevalence of NAFLD in the general European population to be $20-30 \%$ [33]. The characteristics of patients in that study were comparable with those with NAFLD in a recent systematic review of the literature and meta-analysis that included 101 studies [13]. That study reported the European prevalence of NAFLD diagnosed by imaging to be $24 \%$ (95\% CI: $16-34 \%$ ) and diagnosed by blood tests to be 13\% (95\% CI: 4-33\%). Thus, our pooled prevalence in European EHR databases of $1.9 \%$ is at best $\sim 1 / 6$ and more likely only $\sim 1 / 12$ of the estimates based on cohort data. Our estimates of incidence in 2015 ranged from 1.1 to 4.1 per 1000 and are approximately 10 times lower than expected based on cohort studies: 28 (95\% CI: 19-41) per 1000 person-years in Israel and 52 (95\% CI: 28-97) per 1000 in Asia [13].

The prevalence of NAFLD diagnosis has trebled and incidence has doubled over the period of this study. The rising rates of co-morbid conditions such as diabetes and obesity may be responsible for this. Other probable factors include increased awareness among primary-care and non-liver physicians, improved communication of the diagnosis from secondary to primary care, and the increased use of blood tests and imaging to investigate 
common complaints such as abdominal pain or monitoring long-term conditions. Our data do not allow us to test these hypotheses further; however, studies from other groups also suggest that the total number of people developing NAFLD is rising, as is the number of people with NAFLD who develop life-threatening complications [13].

Despite the consistency in overall findings, the differences between the databases are indicative of differing practices. SIDIAP had a relatively large proportion of patients with a history of alcohol abuse (14.1\%), although all databases included at least some NAFLD patients with recorded alcohol abuse. This reflects uncertainty in the community as to whether an individual can have fatty liver disease associated with metabolic syndrome even if they drink alcohol in excess of recommended limits, or indeed have any other cause of chronic liver injury such as viral hepatitis. While clinical trials make very precise distinctions between alcoholic and non-alcoholic fatty liver disease, the reality is that an obese, diabetic and hypertensive patient can consume alcohol in excess of recommended limits and have liver injury. There is no way to distinguish which aetiology is the dominant cause, and so clinicians are quite comfortable with co-existing diagnoses. Indeed, some authors now refer to BAFLD - both alcoholic and fatty liver disease. An alternative explanation may be that specialists making the diagnosis of fatty liver are unaware of the high alcohol use, either because of under-reporting by patients or poor communication from GP practices.

In HSD, prevalence increased over time whereas incidence has decreased in recent years. This can be explained by a relatively stable population in which nearly all patients were enrolled in 2000, see Additional file 1: Figure S3, and remained in the database until December 2015.

Text-mining in IPCI increased the number of NAFLD diagnoses by over eightfold. This suggests that while the diagnosis of NAFLD is being made, GPs are not recording it, despite there being a code for liver steatosis in IPCI. IPCI had the lowest level of ALT recording. A recent survey of Dutch GPs explored attitudes to the importance of NAFLD [34]. Only $47 \%$ of doctors used liver tests in patients with NAFLD and non-invasive scores were never used by $73 \%$ of respondents (we were able to calculate FIB-4 scores in only $27 \%$ in IPCI).

The UK THIN database appears to outlie from the others in several ways. The prevalence of recorded NAFLD in THIN (0.2\%) is much lower than the other databases and markedly lower than that found in a study of almost 700,000 adults in a primary-care EHR study in London, UK $(0.9 \%)$ [35]. Higher rates of alcohol recording in the UK alone are unlikely to account for all this difference. The median ALT was highest in THIN. This may suggest that the diagnosis of NAFLD is more likely to be made in the UK by investigating abnormal liver enzymes than in other territories. However, the data required to calculate FIB- 4 were available in only $11 \%$ of patients in THIN (Additional file 1: Table S3). NAFLD patients in THIN had the highest mean BMI. Moreover, THIN had the highest proportion of NAFLD patients with diabetes or impaired fasting glucose and the highest proportion of NAFLD patients with high-risk FIB-4 scores. Large-scale liver-biopsy-based cross-sectional studies or replication of the current study in cohorts with systematic ascertainment of the component of FIB-4 would be needed to confirm that patients are diagnosed with NAFLD at more advanced stages in the UK compared to other European countries.

\section{Limitations of the study}

When interpreting the data, it is important to consider the following issues. In IPCI, a diagnostic code for NAFLD was not available, therefore we devised an algorithm based on the diagnostic code 'liver steatosis' and excluding excess alcohol consumption. We did not do this for all databases because the IPCI terminology contains only 1073 clinical terms and therefore, general practitioners often utilise the free text to record information with greater precision, whereas the other coding systems contain many more such concepts: ICD9CM contains 40,855 terms, ICD10 contains 13,505 terms and Read Codes contains 347,568 terms [36].

The number of cases of recorded NASH is too small to make meaningful estimates of incidence and prevalence: $2-4 \%$ of patients with NAFLD in THIN and SIDIAP in which NASH was coded. This is far short of the $12.2 \%$ estimated from a US biopsy-based study [37]. This shortfall between coded NASH and the true burden of disease is probably due to the same factors that result in under-recording of NAFLD diagnosis: recognition, referral and coding in primary care, and under-diagnosis or poor communication in secondary care.

It is not possible to verify the accuracy or origin of recorded diagnoses, although the characteristics of the patients derived from the four databases are in keeping with the population one would expect with a NAFLD diagnosis. Some individuals not in this study may have undiagnosed NAFLD. Therefore, our results do not represent the true disease burden in the epidemiological sense, rather they tell us what is actually happening with people who currently have a diagnosis of NAFLD and they can inform the arguments for or against greater action in this area. While we cannot exclude the possibility (however unlikely) that all the other millions of expected NAFLD patients exist in other databases, we do not make any conclusions about people outside this dataset. Although primary-care data contain a large body of 
information, this does not diminish the value of well-phenotyped cohort studies in which NAFLD can be ascertained systematically using standardised screening methods (e.g. measuring liver enzymes or performing ultrasound in all patients). That said, the databases included in this study have been extensively used for research and have been validated for diagnoses other than NAFLD [24, 27, 38].

\section{Conclusions}

Clinical practice is evolving in this emerging field and as yet there are no recommendations to screen formally for NAFLD, even in high risk groups [39, 40]. One school of thought is that if the only available intervention for NAFLD or NASH is lifestyle change, then doctors are already giving such advice to their patients, although the extent to which patients take up such advice varies. However, hepatic steatosis is an independent predictor of diabetes [41, 42] and could, therefore, identify patients who stand to benefit from lifestyle changes to prevent diabetes and hepatic complications. Furthermore, the emerging data suggesting hepatic steatosis is an independent cardiovascular risk factor may be an additional incentive for physicians to increase their awareness of the early stages of NAFLD. At the more severe end of the scale, novel therapies targeted at NASH and fibrosis are already in phase III clinical trials and are expected to be available in the next few years. These may change the treatment paradigm. Therefore, the scale of the health-care challenge posed by NAFLD and its sequelae cannot simply be side-stepped by dismissing NAFLD as pre-disease. Further research is required to quantify the associations of NAFLD with outcomes and to determine whether Wilson's criteria for effective screening can be fulfilled [43], thereby informing the screening debate.

\section{Additional file}

Additional file 1: Table S1. Characteristics of the primary-care databases included in the study. Figure S1. Identification of NAFLD patients in the IPCI database. Table S2. List of codes for the identification of NAFLD and description in ICD9CM, ICD10, Read Codes and SNOMEDCT US terminologies. Table S3. Number and proportion of patients with individual patient characteristic data available. Table S4. Point prevalence ( $95 \% \mathrm{Cl}$ ) of NAFLD and NASH (per 100 persons) on 1 January of each calendar year in four primary-care databases, and pooled across databases. Table S5. One-year period prevalence (95\% CI) of NAFLD (per 100 persons) on 1 January of each calendar year in four primary-care databases. Table S6. Point prevalence (95\% Cl) of NAFLD (per 1000 persons) by age categories and gender on 1 January 2015 in four primary-care databases. Table S7. Incidence estimates (95\% Cl) of NAFLD (per 1000 person-years) by calendar year in four primary-care databases, and pooled estimates across databases. Table S8. Incidence estimates (95\% Cl) of NAFLD (per 1000 person-years) in 2015 by age categories and gender in four primary-care databases. Figure S2. Pooled a prevalence (per 100 persons) and $\mathbf{b}$ incidence (per 1000 person-years) regressed over calendar year by meta-regression. Figure S3. Distribution of entry date for patients in the four databases. (DOCX $203 \mathrm{~kb}$ )

\section{Abbreviations}

ALT: Alanine transaminase; ANOVA: Analysis of variance; AST: Aspartate transaminase; BMI: Body mass index; Cl: Confidence interval; EHR: Electronic health record; EMIF: European Medical Information Framework;

ERC: European Research Council; GP: General practitioner; HSD: Health Search Database; IPCI: Integrated Primary Care Information; NAFLD : Non-alcoholic fatty liver disease; NASH: Non-alcoholic steatohepatitis; NIHR: National Institute for Health Research; SIDIAP: Information System for Research in Primary Care; THIN: The Health Improvement Network; UK: United Kingdom; UMLS: Unified Medical Language System; US: United States

\section{Acknowledgements}

EMIF is a collaboration between industry and academic partners that aims to develop common technical and governance solutions to facilitate access to diverse electronic medical and research data sources. These analyses were supported by the Innovative Medicines Initiative Joint Undertaking under EMIF grant agreement 115372, whose resources include financial contributions from the European Union's Seventh Framework Programme (FP7/2007-2013) and in-kind contributions from European Federation of Pharmaceutical Industries and Associations companies. The authors would like to acknowledge Nicholas Galwey for his advice on the statistical methods, Alba Jene for her administrative support and support during submission to ethical review boards, and Derek Nunez for support during the early protocol design stage.

\section{Funding}

Funding was received from FP7 Ideas under European Research Council (ERC) award 115372. ERC had no role in the design of the study, the collection, analysis, and interpretation of data, or in writing the manuscript. DPA is funded by a National Institute for Health Research (NIHR) Clinician Scientist award (CS-2013-13-012). This article presents independent research funded by the $\mathrm{NIHR}$. The views expressed are those of the authors and not necessarily those of the National Health Service in the UK, the NIHR or the Department of Health. This work was partially supported by the NIHR Biomedical Research Centre, Oxford. WA is in receipt of a Medical Research Council New Investigator Award.

\section{Availability of data and materials}

This work uses data provided by patients and collected by the different health-care systems involved as part of their care and support. All data relevant to the study purpose are within the paper and its supporting files. Original individual-level data are in the custody of local partners, and access depends on local governance rules. Local restrictions on publicly sharing original study data may vary case-by-case and depend on the institutional review board, the ethics committee or the law. Further information on data requests and access should be sent individually to the authors of this paper who are responsible for the data provided by the relevant organisations: SIDIAP (tduarte@idiapjgol.org), HSD (lapi.francesco@simg.it), THIN (d.ansell@bham.ac.uk) and IPČ́l (j.vanderlei@erasmusmc.nl).

\section{Authors' contributions}

MA, AKL, PE, SK, DW, NS and WA designed the study. TDS and PA undertook the semantic harmonisation. PR was responsible for the data transformation and federated data analysis. MA and JFB analysed the data. MA, NS and WA wrote the manuscript. All authors interpreted the results, edited the manuscript and gave approval for submission.

\section{Ethics approval and consent to participate}

We followed local data laws in all four territories from which the data were obtained. In all countries, specific ethical approval was not required for this study as it used anonymised data. However, approval was sought and obtained from the scientific research committee for THIN, the IPCI Governing Board (reference 2015/18), the SIDIAP Ethics Committee (reference P15/167) and the scientific committee of the Italian College of General Practitioners and Primary Care.

\section{Consent for publication}

Not applicable.

\section{Competing interests}

MA was contracted to work at, and JFB, PE, SK and DW are employees of, GlaxoSmithKline, which has conducted clinical research including trials of therapeutic agents in NAFLD. AKL is an employee of Pfizer, which is 
conducting clinical research including trials of therapeutic agents in NAFLD DPA has received unrestricted research grants from UCB, Amgen and Servier, and consultancy fees (paid to his department or research group) from UCB Pharma. DA has provided consultancy and advice to many pharmaceutical companies on undertaking outcome studies using real-world evidence. FL has provided consultancy tor AlfaSigma, Bayer and Abbvie. PE and SK are employees and stockholders of GlaxoSmithKline. NS has consulted for Boehringer Ingelheim, Eli Lilly, Novo Nordisk and Janssen, and has received grants from Astrazeneca and BI. WA is a consultant and has delivered sponsored lectures to UCB Pharma, Gilead, Intercept and Medimmune. TDS has no conflicts of interest to declare.

\section{Publisher's Note}

Springer Nature remains neutral with regard to jurisdictional claims in published maps and institutional affiliations.

\section{Author details}

${ }^{1}$ GlaxoSmithKline, London, UK. ${ }^{2}$ Worldwide Research and Development, Pfizer, Connecticut, USA. ${ }^{3}$ Erasmus Universitair Medisch Centrum, Rotterdam, The Netherlands. ${ }^{4}$ Fundació Institut Universitari per a la Recerca a I'Atenció Primària de Salut Jordi Gol i Gurina, Barcelona, Spain. ${ }^{5}$ Centre for Statistics in Medicine, NDORMS, University of Oxford, Oxford, UK. ${ }^{6}$ Quintile IMS, London, UK. ${ }^{7}$ Health Search, Italian College of General Practitioners and Primary Care, Florence, Italy. ${ }^{8}$ Harvard Medical School, Harvard, Boston, MA, USA. ${ }^{9}$ University of Glasgow, BHF Glasgow Cardiovascular Research Centre, Glasgow, UK. ${ }^{10}$ Barts Liver Centre, Blizard Institute, Queen Mary, University of London, London, UK.

Received: 29 March 2018 Accepted: 19 June 2018

Published online: 13 August 2018

\section{References}

1. Sattar N, Forrest E, Preiss D. Non-alcoholic fatty liver disease. BMJ. 2014;349:94596.

2. Tai FW, Syn WK, Alazawi W. Practical approach to non-alcoholic fatty liver disease in patients with diabetes. Diabet Med. 2015;32(9):1121-33.

3. Mantovani $\mathrm{A}$, et al. Nonalcoholic fatty liver disease and risk of incident type 2 diabetes: a meta-analysis. Diabetes Care. 2018:41(2):372-82.

4. Mantovani A, et al. Nonalcoholic fatty liver disease increases risk of incident chronic kidney disease: a systematic review and meta-analysis. Metabolism. 2018;79:64-76.

5. Targher $\mathrm{G}$, et al. Non-alcoholic fatty liver disease and risk of incident cardiovascular disease: a meta-analysis. J Hepatol. 2016;65(3):589-600.

6. Ekstedt $M$, et al. Fibrosis stage is the strongest predictor for disease-specific mortality in NAFLD after up to 33 years of follow-up. Hepatology. 2015; 61(5):1547-54

7. Söderberg C, et al. Decreased survival of subjects with elevated liver function tests during a 28-year follow-up. Hepatology. 2010;51(2):595-602.

8. Sanna C, et al. Non-alcoholic fatty liver disease and extra-hepatic cancers. Int J Mol Sci. 2016;17(5):171.

9. Lonardo A, et al. Epidemiological modifiers of non-alcoholic fatty liver disease: focus on high-risk groups. Dig Liver Dis. 2015;47(12):997-1006.

10. Targher G, Day CP, Bonora E. Risk of cardiovascular disease in patients with nonalcoholic fatty liver disease. N Engl J Med. 2010;363(14):1341-50.

11. Targher G, Lonardo A, Byrne CD. Nonalcoholic fatty liver disease and chronic vascular complications of diabetes mellitus. Nat Rev Endocrinol. 2018;14(2):99-114.

12. Allen $\mathrm{AM}$, et al. Nonalcoholic fatty liver disease incidence and impact on metabolic burden and death: a 20 year-community study. Hepatology. 2016;64(6):2165-72.

13. Younossi ZM, et al. Global epidemiology of nonalcoholic fatty liver diseasemeta-analytic assessment of prevalence, incidence, and outcomes. Hepatology. 2016;64(1):73-84.

14. Williamson RM, et al. Prevalence of and risk factors for hepatic steatosis and nonalcoholic fatty liver disease in people with type 2 diabetes: the Edinburgh type 2 diabetes study. Diabetes Care. 2011;34(5):1139-44

15. Vernon G, Baranova A, Younossi ZM. Systematic review: the epidemiology and natural history of non-alcoholic fatty liver disease and non-alcoholic steatohepatitis in adults. Aliment Pharmacol Ther. 2011;34(3):274-85.

16. Bedossa $P$, et al. Systematic review of bariatric surgery liver biopsies clarifies the natural history of liver disease in patients with severe obesity. Gut. 2017; 66(9):1688-96.
17. Zezos P, Renner EL. Liver transplantation and non-alcoholic fatty liver disease. World J Gastroenterol. 2014;20(42):15532-8.

18. Rinella ME. Screening for nonalcoholic fatty liver disease in patients with atherosclerotic coronary disease?-in principle yes, in practice not yet. Hepatology. 2016;63(3):688-90.

19. Wong W, Chalasani N. Not routine screening, but vigilance for chronic liver disease in patients with type 2 diabetes. J Hepatol. 2016;64(6):1211-3.

20. Booth $\mathrm{H}$, et al. Incidence of type 2 diabetes after bariatric surgery: populationbased matched cohort study. Lancet Diabetes Endocrinol. 2014;2(12):963-8.

21. Farmer RD, et al. Population-based study of risk of venous thromboembolism associated with various oral contraceptives. Lancet. 1997; 349(9045):83-8.

22. Hobbs FDR, et al. Clinical workload in UK primary care: a retrospective analysis of 100 million consultations in England, 2007-14. Lancet. 2016; 387(10035):2323-30.

23. Kringos $D$, et al. The strength of primary care in Europe: an international comparative study. Br J Gen Pract. 2013;63(616):e742-50.

24. Gini $R$, et al. Chronic disease prevalence from Italian administrative databases in the VALORE project: a validation through comparison of population estimates with general practice databases and national survey. BMC Public Health. 2013;13:15.

25. Vlug $A E$, et al. Postmarketing surveillance based on electronic patient records: the IPCI project. Methods Inf Med. 1999;38(4-5):339-44.

26. Blak BT, et al. Generalisability of the health improvement network (THIN) database: demographics, chronic disease prevalence and mortality rates. Inform Prim Care. 2011:19(4):251-5.

27. Garcia-Gil Mdel M, et al. Construction and validation of a scoring system for the selection of high-quality data in a Spanish population primary care database (SIDIAP). Inform Prim Care. 2011;19(3):135-45.

28. Avillach P, et al. Harmonization process for the identification of medical events in eight European healthcare databases: the experience from the EUADR project. J Am Med Inform Assoc. 2013;20(1):184-92.

29. Sterling RK, et al. Development of a simple noninvasive index to predict significant fibrosis in patients with HIV/HCV coinfection. Hepatology. 2006; 43(6):1317-25.

30. Shah AG, et al. Comparison of noninvasive markers of fibrosis in patients with nonalcoholic fatty liver disease. Clin Gastroenterol Hepatol. 2009;7(10): 1104-12.

31. Coloma PM, et al. Combining electronic healthcare databases in Europe to allow for large-scale drug safety monitoring: the EU-ADR project. Pharmacoepidemiol Drug Saf. 2011;20(1):1-11.

32. Nascimbeni $F$, et al. From NAFLD in clinical practice to answers from guidelines. J Hepatol. 2013;59(4):859-71.

33. Loomba R, Sanyal AJ. The global NAFLD epidemic. Nat Rev Gastroenterol Hepatol. 2013;10(11):686-90.

34. van Asten $M$, et al. The increasing burden of NAFLD fibrosis in the genera population: time to bridge the gap between hepatologists and primary care. Hepatology. 2017;65(3):1078.

35. Alazawi $W$, et al. Ethnicity and the diagnosis gap in liver disease: a population-based study. Br J Gen Pract. 2014;64(628):e694-702.

36. Medicine, N.L.o. Unified Medical Language System. 2017; Available from: https://www.nlm.nih.gov/research/umls/sourcereleasedocs/mrsabfields.html.

37. Williams CD, et al. Prevalence of nonalcoholic fatty liver disease and nonalcoholic steatohepatitis among a largely middle-aged population utilizing ultrasound and liver biopsy: a prospective study. Gastroenterology. 2011;140(1):124-31.

38. Coloma PM, et al. Identification of acute myocardial infarction from electronic healthcare records using different disease coding systems: a validation study in three European countries. BMJ Open. 2013;3(6):e002862.

39. Chalasani N, et al. The diagnosis and management of nonalcoholic fatty liver disease: practice guidance from the American Association for the Study of Liver Diseases. Hepatology. 2018;67(1):328-57.

40. European Association for the Study of the, L., D. European Association for the Study of, and O. European Association for the Study of. EASL-EASDEASO clinical practice guidelines for the management of non-alcoholic fatty liver disease. J Hepatol. 2016;64(6):1388-402.

41. Sung KC, Kim SH. Interrelationship between fatty liver and insulin resistance in the development of type 2 diabetes. J Clin Endocrinol Metab. 2011;96(4):1093-7.

42. Zelber-Sagi S, et al. Non-alcoholic fatty liver disease independently predicts prediabetes during a 7-year prospective follow-up. Liver Int. 2013;33(9):1406-12.

43. Wilson JMG, Jungner Gja. Principles and practice of screening for disease [by] J. M. G. Wilson [and] G. Jungner. Geneva: World Health Organization; 1968. 